\title{
FSH antibody prevents obesity and loss of bone
}

...mice given
the antibody
did not
accumulate
adipose
tissue after
undergoing
ovariectomy or
consuming
a HFD

Levels of follicle-stimulating hormone (FSH) rise during the peri-menopausal transition; women often gain visceral fat and experience bone loss at this time, which puts them at increased risk of fractures, type 2 diabetes mellitus and cardiovascular disease. Current treatment strategies do not adequately address these risks. A new paper published in Nature suggests that a FSH antibody might be able to simultaneously prevent loss of bone and accumulation of adipose tissue.

"In prior studies, we had shown that the pituitary hormone FSH, which was thought solely to affect
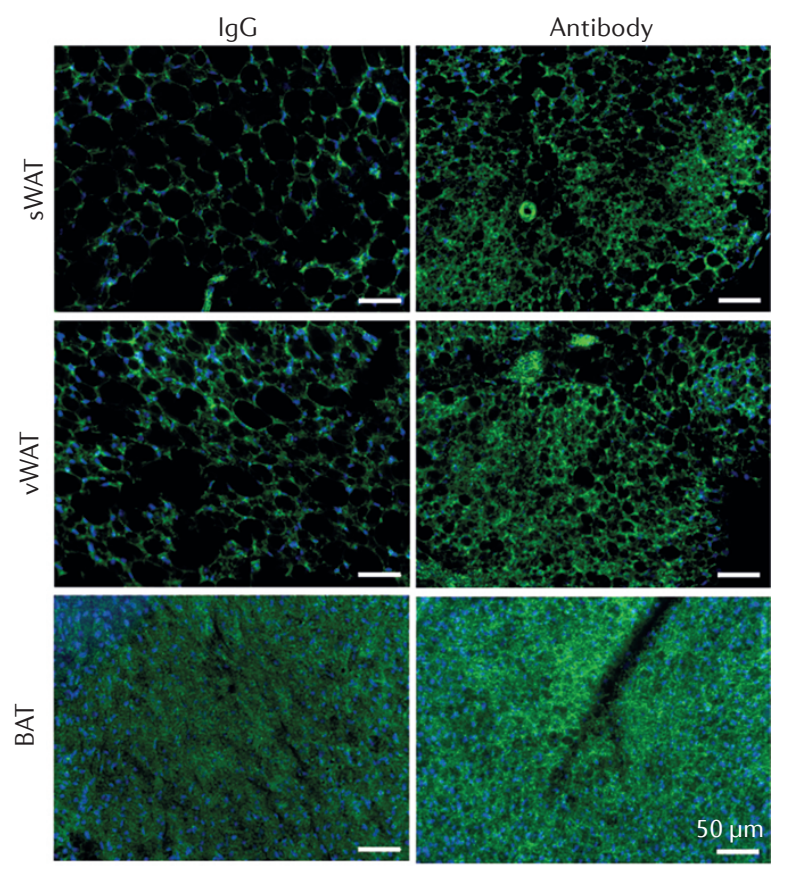

Fluorescence indicative of mitochondrial biogenesis in brown adipose tissue (BAT), visceral white adipose tissue (VWAT) and subcutaneous WAT (sWAT) of mice after 2 weeks of treatment with immunoglobulin G $(\mathrm{lgG})$ or antibody. Permission obtained from Liu, P. et al. Nature http://dx.doi.org/10.1038/nature22342 (2017), Macmillan Publishers Limited. oestrogen secretion, is a direct regulator of bone mass and that a polyclonal antibody that blocks FSH reduces bone loss after ovariectomy in mice," explains corresponding author Mone Zaidi. To build on these findings, the researchers investigated whether targeting FSH could also reduce the accumulation of visceral fat.

Zaidi and colleagues used male and female mice fed a high-fat diet (HFD) or normal chow, as well as ovariectomized mice fed normal chow. The mice were either administered with goat immunoglobulin G (IgG) as the control or the polyclonal antibody $(200 \mu \mathrm{g}$ per day), which targeted a 13-amino-acid sequence in the $\beta$-subunit of FSH (FSH $\beta$ ). Body fat was then measured using various methods, including dual energy X-ray absorptiometry and quantitative nuclear magnetic resonance. The effects on beiging were determined using immunocytochemistry for UCP1 (a marker of beiging) and by using quantitative PCR to measure Ucp1 expression in white and brown adipose tissue. Studies in metabolic cages were used to estimate thermogenesis.

The investigators confirmed that the antibody prevented bone loss in ovariectomized mice. However, they also found that mice given the antibody did not accumulate adipose tissue after undergoing ovariectomy or consuming a HFD. "The antibody also caused profound beiging and activated brown adipose tissue to enhance thermogenesis," explains Zaidi. "These actions resulted from the specific binding of the antibody to FSH $\beta$ to block its action."
Furthermore, male mice deficient in the $\mathrm{FSH}$ receptor ( $\mathrm{Fshr} \mathrm{r}^{+/-}$mice) had a similar phenotype to the wildtype mice that received the antibody after ovariectomy or a HFD. This finding suggests that FSH could be an important regulator of fat mass. No changes in any of the measured parameters were seen when the $\mathrm{Fshr}^{+/-}$mice were given the antibody, which supports the idea that the antibody suppresses the FSH signalling axis in vivo.

Finally, the researchers developed a monoclonal antibody (called Hf2) that targets a human $\mathrm{FSH} \beta$ epitope. Hf2 was found to bind to mouse and human FSH $\beta$. In addition, mice fed a HFD that were injected with Hf2 displayed a similar phenotype to mice administered with the polyclonal antibody.

"These paradigm-shifting findings provide the novel opportunity to treat bone loss and visceral fat accumulation simultaneously in women," concludes Zaidi. "Notably, as serum levels of FSH increase in the 2-3 years before the menopause, and bone loss and visceral fat gain both precede menopause, there is a clear window of opportunity to maintain health in this group." Zaidi and co-workers hope that a humanized version of Hf 2 could be used to reduce visceral and subcutaneous fat in both women and men, which would be beneficial for preventing type 2 diabetes mellitus and managing cardiovascular risk.

\section{Claire Greenhill}

ORIGINAL ARTICLE Liu, P. et al. Blocking FSH induces thermogenic adipose tissue and reduces body fat. Nature http://dx.doi.org/10.1038/ nature22342 (2017) 